\title{
Associação de paralisia diafragmática bilateral e paralisia da cintura escapular após correção de aneurisma de aorta: relato de caso
}

\author{
Luiz Marcelo Sá MALBOUISSON*, Denise PERES**, Sérgio AYAMA**, \\ Maria José Carvalho CARMONA*, José Otávio Costa AULER Jr.*
}

RBCCV 44205-544

\begin{abstract}
Malbouisson L M S, Peres D, Ayama S, Carmona M J C, Auler Jr. J O C. - Associação de paralisia diafragmática bilateral e paralisia da cintura escapular após correção de aneurisma de aorta: relato de caso. Rev Bras Cir Cardiovasc 2001; 16(2): 171-5.
\end{abstract}

\begin{abstract}
RESUMO: A associação de paralisia diafragmática bilateral e paralisia da cintura escapular é uma complicação rara após correção de aneurisma de aorta ascendente. Esta associação leva à uma forma grave de insuficiência respiratória cujo sucesso do desmame da ventilação mecânica é dependente da capacidade da musculatura respiratória remanescente em compensar o trabalho respiratório excedente. Fisioterapia respiratória intensiva com treinamento da musculatura, correção dos distúrbios metabólicos, tratamento das infecções respiratórias associadas à ventilação mecânica e otimização do suporte nutricional foram fundamentais para o sucesso do desmame.
\end{abstract}

DESCRITORES: Paralisia respiratória, etiologia. Procedimentos cirúrgicos cardíacos, efeitos adversos. Aneurisma aórtico, cirurgia. Nervo frênico. Escápula. Plexo braquial. Paralisia respiratória, terapia.

\section{INTRODUÇÃO}

A paralisia diafragmática bilateral é uma das complicações respiratórias mais graves observadas no período pós-operatório de cirurgia cardiovascular. Os pacientes que desenvolvem esta complicação necessitam de suporte ventilatório prolongado e apresentam alto risco para o desenvolvimento de pneumonias associadas à ventilação(1). Na presença de paralisia diafragmática bilateral, o desmame da ventilação mecânica é de- pendente da integridade dos músculos acessórios da respiração, que devem compensar a perda funcional do diafragma. A associação de paralisia frênica bilateral e paralisia da cintura escapular representa complicação pós-operatória rara e desafio terapêutico, uma vez que parte da musculatura acessória está não funcionante. Neste relato, apresentamos a associação de paralisia frênica bilateral e paralisia da cintura escapular em paciente submetida à correção de aneurisma de aorta ascendente e suas implicações no desmame da ventilação mecânica e na terapêutica.

\footnotetext{
Trabalho realizado no Instituto do Coração do Hospital das Clínicas da Faculdade Medicina da Universidade de São Paulo. São Paulo, SP, Brasil. Recebido para publicação em janeiro de 2001.

* Do Serviço de Anestesiologia e Terapia Intensiva Cirúrgica do Instituto do Coração do Hospital das Clínicas da Faculdade de Medicina da Universidade de São Paulo.

** Do Serviço de Fisioterapia do Instituto do Coração do Hospital das Clínicas da Faculdade de Medicina da Universidade da Faculdade de Medicina de São Paulo.

Endereço para correspondência: Luiz Marcelo Sá Malbouisson. Instituto do Coração. Serviço de Anestesiologia. Av. Dr. Enéas de Carvalho Aguiar, 44. São Paulo, SP, Brasil. CEP 05403-000. Tel.: (11) 3069-5232. e-mail: carmona@incor.usp.br
} 


\section{RELATO DE CASO}

Paciente M. L. M., 43 anos, sexo feminino, com diagnóstico de aneurisma de aorta tipo $\mathrm{A}$, com diâmetro máximo de $65 \mathrm{~mm}$, em tratamento clínico com betabloquedor e vasodilatadores orais. Deu entrada no Serviço de Emergência do Instituto do Coração do HCFMUSP apresentando quadro de dor precordial irradiada para dorso, náuseas e sudorese intensa. Encontrava-se hemodinamicamente estável à admissão e os pulsos periféricos estavam presentes ao exame clínico. Tomografia computadorizada de tórax evidenciou aumento do diâmetro máximo do aneurisma para $72 \mathrm{~mm}$ (Figura 1), sem sinais sugestivos de dissecção, sendo indicada operação após estabilização clínica do quadro. A paciente apresentava como antecedentes cirúrgicos lesão de artéria braquial direita, sendo feito enxerto braquiobraquial de veia safena sem sucesso.

A operação para correção do aneurisma de aorta foi realizada nove dias após a admissão da paciente ao hospital. O procedimento cirúrgico foi iniciado após anestesia geral e canulação artériovenosa femoral esquerda. Foi instituído hipotermia profunda à $17^{\circ} \mathrm{C}$, seguida de parada circulatória total. Foram utilizadas cardioplegia cristalóide tipo Saint Thomaz e retroperfusão cerebral. A aorta ascendente foi substituída por prótese de Dacron número 30 , preservando-se a valva aórtica. $\mathrm{O}$ tempo de parada circulatória total foi de 25 minutos, com tempo de circulação extracorpórea de 2 horas e 10 minutos. Não houve intercorrências durante a operação.

A paciente foi admitida à Unidade de Terapia Intensiva hemodinamicamente estável, recebendo dobutamina e nitroprussiato de sódio. O suporte ventilatório foi ventilação mandatória sincronizada intermitente (VMSI) com pressão controlada de 25 $\mathrm{cmH}_{2} \mathrm{O}$, freqüência respiratória (FR) de $12 \mathrm{ipm}$, pressão de suporte (PS) de $20 \mathrm{cmH}_{2} \mathrm{O}$, pressão positiva ao final da inspiração (PEEP) de $8 \mathrm{cmH}_{2} \mathrm{O}$ e fração inspirada de oxigênio $\left(\mathrm{FiO}_{2}\right)$ de 0.6. Devido à agitação psicomotora ao despertar da anestesia, a paciente foi sedada com propofol em infusão contínua e mantida intubada por 24 horas. No 1 dia pós-operatório (DPO), observou-se diminuição dos murmúrios vesiculares à ausculta pulmonar e elevação da cúpula diafragmática direita à radiografia de tórax. O desmame da ventilação mecânica foi realizado sem intercorrências e a paciente foi extubada com PS de $8 \mathrm{cmH}_{2} \mathrm{O}$, PEEP de $10 \mathrm{cmH}_{2} \mathrm{O}$ e $\mathrm{FiO}_{2}$ 0.4 , mantendo volume corrente expirado de $400 \mathrm{ml}$ e oxigenação satisfatória. Após a extubação, foi instituída ventilação não invasiva (VNI) com PS 20 $\mathrm{cmH}_{2} \mathrm{O}$, PEEP $20 \mathrm{cmH}_{2} \mathrm{O}$ e $\mathrm{FiO}_{2}$ 0.4.

No 2 DPO, observou-se piora do padrão respiratório, com respiração paradoxal após inter-

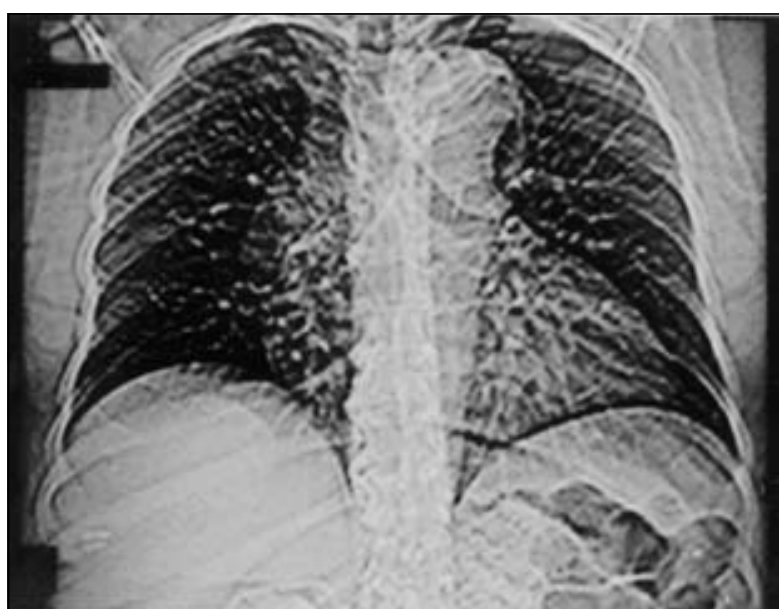

Fig. 1 - Reconstrução sagital de imagens obtidas através de tomografia computadorizada durante a avaliação inicial.

rupção da $\mathrm{VNI}$, acompanhada de rebaixamento do nível de consciência, havendo necessidade de reintubação. Instituiu-se ventilação mecânica com PEEP em $10 \mathrm{cmH}_{2} \mathrm{O}$ e PS em $20 \mathrm{cmH}_{2} \mathrm{O}, \mathrm{FiO}_{2}$ de 0.4 e iniciado plano de desmame da PS em $1 \mathrm{cmH}_{2} \mathrm{O}$ a cada 12 horas. Radiografia torácica mostrava elevação bilateral das cúpulas frênicas sugerindo paralisia frênica (Figura 2). Ultrassonografia de tórax e tomografia de tórax confirmaram o diagnóstico (Figura 3). Manobras de recrutamento alveolar em ventilação espontânea com PEEP de $20 \mathrm{cmH}_{2} \mathrm{O}$ foram realizadas a cada 3 horas. No 4 DPO, a paciente apresentou episódio de broncoaspiração, que evoluiu para pneumonia, sendo tratada com ceftriaxone e clindamicina. No 9 DPO, com os parâmetros de ventilação mecânica baixos, foi realizada nova tentativa de extubação. Após algumas horas, a paciente evoluiu com respiração paradoxal, hipoxêmia e hipercapnia, sendo reintubada. Traqueostomia percutânea à beira do leito foi realizada no 11 DPO. No 12 DPO, foi observado ao exame clínico ausência de movimentos da cintura escapular, ombros, cotovelos e de alguns grupos musculares cervicais. A avaliação neurológica evidenciou paralisia braquial proximal bilateral e da cintura escapular, sendo feito hipótese diagnóstica de lesão isquêmica da medula. Realizado ressonância nuclear magnética que confirmou o diagnóstico. No 14 DPO, a paciente fazia pressão inspiratória máxima $\left(\mathrm{PI}_{\max }\right)$ de $32 \mathrm{cmH}_{2} \mathrm{O}$, pressão expiratória máxima $\left(\mathrm{PE}_{\max }\right)$ de $36 \mathrm{cmH}_{2} \mathrm{O}$ e foi iniciado treinamento muscular com carga de $-13 \mathrm{cmH}_{2} \mathrm{O}$, que representava $40 \%$ do valor de $\mathrm{PI}_{\max }$ obtido. Episódios de pneumonia associada à ventilação mecânica contribuíram para a falência das tentativas de desmame da ventilação mecânica. No 40 DPO, repetiuse ultra-sonografia torácica que constando-se au- 


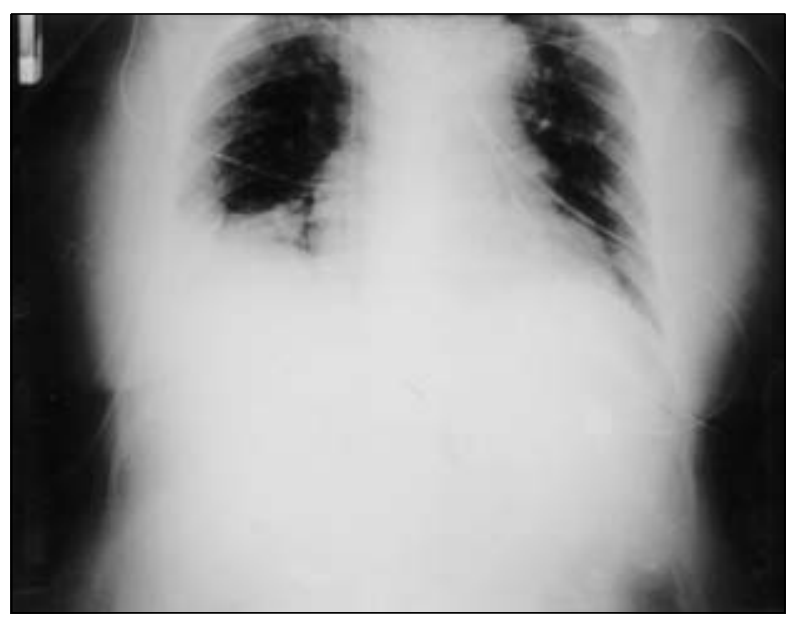

Fig. 2 - Radiografia de tórax obtida na Unidade de Terapia Intensiva, mostrando elevação bilateral das cúpulas frênicas.

sência de mobilidade diafragmática bilateral em ventilação espontânea tentando-se, então, ventilação com pressão negativa, com pressão inspiratória de $-20 \mathrm{cmH}_{2} \mathrm{O}$, tempo inspiratório de 2 segundos e freqüência de 12 IPM. A ventilação por pressão negativa foi suspensa no 43 DPO devido à inadaptação da paciente à ventilação. No 47 DPO, detectou-se aumento da área cardíaca à radiografia de tórax, sendo realizada tomografia de tórax que evidenciou coleção retroesternal, presença de derrame pericárdico moderado em região posterior $\mathrm{e}$ atelectasias basais bilaterais. No 49 DPO, foi iniciado tubo T com válvula de PEEP de $10 \mathrm{~cm} \mathrm{H}_{2} \mathrm{O}$ por períodos de 15 minutos durante a manhã e a tarde. Instituiu-se controle diário de peso e balanço hídrico negativo com diuréticos. Os períodos de nebulização em tubo $\mathrm{T}$ foram progressivamente aumentados.

No 68 DPO, a paciente evoluiu com instabilidade hemodinâmica, oligúria e desconforto respiratório durante a nebulização, sendo realizado um ecocardiograma que evidenciou derrame pericárdio importante com sinais de restrição, realizando-se drenagem pericárdica. Devido às múltiplas falências no desmame da ventilação mecânica, a paciente começou à evoluir com depressão. Foi iniciado acompanhamento psicoterápico e antidepressivos orais. Novamente tentou-se nebulização em tubo $T$ com válvula de PEEP durante 15 minutos pela manhã e pela tarde no 73 DPO, a $\mathrm{PI}_{\max }$ era $-20 \mathrm{cmH}_{2} \mathrm{O}$ e a $P E_{\max }$ de $+30 \mathrm{cmH}_{2} \mathrm{O}$. No 81 DPO, conseguiuse tempo total de nebulização de 4 horas e 30 minutos com $\mathrm{PI}_{\max }$ de $-20 \mathrm{cmH}_{2} \mathrm{O}$ e $\mathrm{PE}_{\max }$ de +42 $\mathrm{cmH}_{2} \mathrm{O}$. No 90 DPO, a paciente manteve-se em nebulização por 24 horas consecutivas, com $\mathrm{PI}_{\max }$ de $-20 \mathrm{cmH}_{2} \mathrm{O}$ e $\mathrm{PE}_{\max }$ de $+68 \mathrm{cmH}_{2} \mathrm{O}$ ao final das 24 horas. A cânula de traqueostomia foi trocada por

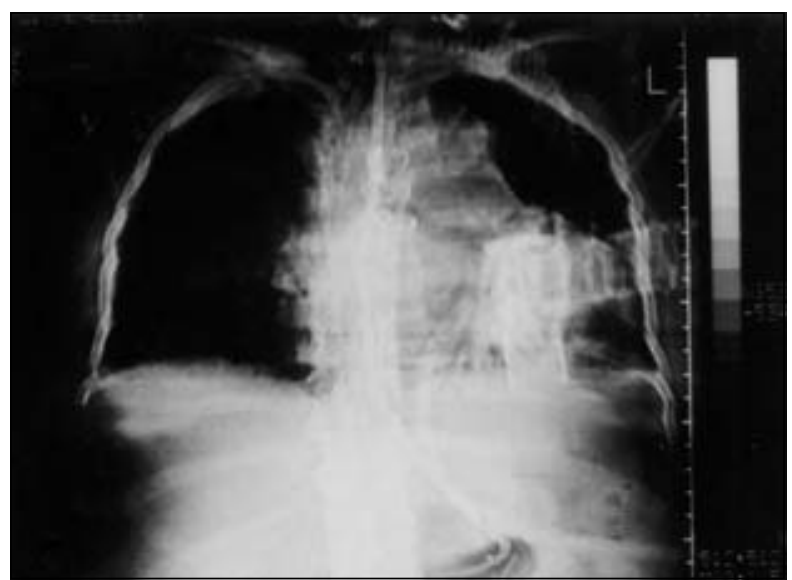

Fig. 3 - Reconstrução sagital de imagens obtidas através de tomografia computadorizada, mostrando elevação e retificação das cúpulas frênicas, sugerindo paralisia frênica bilateral.

cânula metálica no 96 DPO. No 101 DPO foi retirada a cânula de traqueostomia e ocluído o traqueostoma com curativo. A paciente recebeu alta da Unidade de Terapia Intensiva no 104 DPO. Na enfermaria, foi repetido ultra-sonografia de tórax que evidenciou paralisia frênica bilateral. No 111 DPO, a paciente recebeu alta hospitalar mantendo a paralisia frênica e da cintura escapular, mas com ventilação satisfatória.

\section{COMENTÁRIOS}

A paralisia frênica bilateral é complicação freqüentemente observada no pós-operatório de cirurgia cardiovascular (2-6), habitualmente secundária à disfunção ou lesão do nervo frênico ${ }^{(7)}$. Caracterizase por insuficiência respiratória hipercapnica de difícil manejo terapêutico e de desmame difícil do ventilador mecânico ${ }^{(6,8)}$. A fisiopatologia da disfunção frênica no pós operatório de cirurgia cardiovascular é diversa sendo descritos: circulação extracorpórea com a utilização de hipotermia sistêmica profunda $(3,9)$, hipotermia tópica no saco pericárdico ${ }^{(2)}$ e manipulação cirúrgica (10) Nos pacientes que desenvolvem esta complicação, o sucesso do desmame da ventilação mecânica é primariamente dependente da capacidade da musculatura acessória em compensar o trabalho respiratório normalmente realizado pelo diafragma. Contudo, no caso descrito acima, houve também uma perda de força na musculatura proximal dos membros superiores, ausência de contratilidade dos músculos escalenos, grande peitoral e trapézio. Em vista do quadro clínico e do procedimento cirúrgico realizado, suspeitou-se de isquemia medular cérvico-torácica, confirmada através de ressonância 
nuclear magnética. A hipótese de isquemia medular cérvico-torácica explicaria todo o quadro clínico, uma vez que a inervação motora dos músculos acometidos encontra-se no território onde foram observadas lesões sugestivas de isquemia medular através da ressonância nuclear magnética. Entretanto, neste caso não foi possível excluir a participação de outros mecanismos como hipotermia e manipulação cirúrgica na gênese da disfunção frênica. A associação de paralisia diafragmática bilateral e da cintura escapular, incluindo alguns músculos acessórios da respiração, determina como descrevemos uma forma grave de insuficiência respiratória hipercapnica. $O$ tratamento consiste no suporte ventilatório e treinamento muscular até a adaptação da musculatura respiratória remanescente ao trabalho respiratório excedente, e prevenção das complicações secundárias ao suporte ventilatório prolongado que possam contribuir com aumento do trabalho respiratório.

No caso apresentado acima, o trabalho respiratório deveria ser realizado pela musculatura intercostal não afetada, pelo esternocleidomastoídeo e pela musculatura abdominal. A avaliação da pressão inspiratória máxima revelou uma diminuição importante de força da musculatura respiratória remanescente, com valores maiores que - 30 $\mathrm{cmH}_{2} \mathrm{O}$, preditivos de falência de desmame do ventilador mecânico $(11,12)$. Todavia, nenhum índice preditivo de desmame é validado em pacientes com paralisia frênica. Em vista da diminuição da força muscular, o tratamento da paciente foi direcionado para a diminuição do trabalho respiratório. Parte do tratamento baseia-se no suporte nutricional. Os lipídios devem ser preferidos e passam a ser $40 \%$ das calorias não protéicas, com intuito de diminuirse a produção de $\mathrm{CO}_{2}$. Avaliações dos níveis séricos de fósforo, magnésio e cálcio foram realizadas a cada 3 dias durante a sua permanência na Unidade de Terapia Intensiva, para prevenir a fadiga mus- cular induzida por distúrbios hidroeletrolíticos (1315). Os quadros de traqueobronquites e pneumonias associadas à ventilação mecânica foram tratados precocemente para prevenir aumento do trabalho respiratório secundário à hipersecreção pulmonar. O balanço hídrico foi mantido negativo para diminuir o trabalho respiratório secundário à congestão pulmonar e realizado treinamento intensivo da musculatura respiratória remanescente com resistores de carga linear com intuito de aumentar o limiar de fadiga.

É importante ressaltar a utilização de ventilação com pressão negativa através de colete torácico. Esta modalidade ventilatória propicia condições para recuperação da função muscular, sem os riscos inerentes à ventilação por pressão positiva (16), porém a paciente não conseguiu adaptar-se ao método. Após 94 dias na UTI, foi possível descontinuar o suporte ventilatório, sugerindo que a musculatura respiratória remanescente adaptou-se ao trabalho respiratório excedente. Outras possibilidades terapêuticas têm sido empregadas em pacientes adultos com paralisia frênica bilateral como plicatura diafragmática e estimulação elétrica do músculo ${ }^{(17,18)}$, contudo, a nossa experiência com estas técnicas é pequena.

Em conclusão, neste relato, descrevemos um caso de associação de paralisia diafragmática e paralisia da cintura escapular no pós-operatório de operação após correção de aneurisma de aorta ascendente. Não foi observado durante a pesquisa de literatura qualquer caso semelhante. A disfunção diafragmática associada à paralisia da cintura escapular determina uma forma grave insuficiência respiratória, de difícil tratamento, cujo sucesso depende da adaptação da musculatura remanescente à sobrecarga de trabalho respiratório.

Malbouisson L M S, Peres D, Ayama S, Carmona M J C, Auler Jr. J O C. - Association between bilateral diaphragmatic paralysis and scapular girdle paralysis after aortic aneurysm correction: case report. Rev Bras Cir Cardiovasc 2001; 16(2): 171-5.

ABSTRACT: The association of bilateral diaphragmatic paralysis and scapular girdle paralysis is a rare complication after aortic aneurysm correction. Such a complication induces to a severe form of hypercapnic respiratory failure in which the success of weaning from mechanical ventilation depends on the capacity of remaining respiratory muscles to compensate extra respiratory work load. Intensive respiratory physiotherapy with muscle training, hydroelectrolityc inbalances correction, treatment of ventilation associated infections and a well planned nutritional support were essential to the successful weaning of mechanical ventilation.

DESCRIPTORS: Respiratory paralysis, etiology. Cardiac surgical procedures, adverse effects. Aortic aneurysm, surgery. Phrenic nerve. Scapula. Brachial plexus. Respiratory paralysis, therapy. 


\section{REFERÊNCIAS BIBLIOGRÁFICAS}

1 van Onna I E, Metz R, Jekel L, Woolley S R, van de Wal $\mathrm{HJ}$ - Post cardiac surgery phrenic nerve palsy: value of plication and potential for recovery. Eur $J$ Cardiothorac Surg 1998; 14: 179-84.

2 Efthimiou J, Butler J, Woodham C, Benson M K, Westaby $S$ - Diaphragm paralysis following cardiac surgery: role of phrenic nerve cold injury. Ann Thorac Surg 1991; 52: 1005-8.

3 Maccherini M, Davoli G, Sani G et al. - Warm heart surgery eliminates diaphragmatic paralysis. J Card Surg 1995; 10: 257-61.

4 Efthimiou J, Butler J, Benson M K, Westaby S - Bilateral diaphragm paralysis after cardiac surgery with topical hypothermia. Thorax 1991; 46: 351-4.

5 Gordon P C, Bateman E D, Linton D M - Biltateral phrenic nerve palsy following cardiac surgery in a diabetic patient. Anaesth Intensive Care 1992; 20: 511-4.

6 Tripp H F \& Bolton J W - Phrenic nerve injury following cardiac surgery: a review. J Card Surg 1998; 13: 218-23.

7 Wilcox P G \& Pardy R L - Diaphragmatic weakness and paralysis. Lung 1989; 167: 323-41.

8 Lin M C, Liaw M Y, Huang C C, Chuang M L, Tsai Y H - Bilateral diaphragmatic paralysis - a rare of acute respiratory failure managed with nasal mask bilevel positive airway pressure (BiPAP) ventilation. Eur Respir J 1997; 10: 1922-4.

9 Mills G H, Khan Z P, Moxham J, Desai J, Forsyth A, Ponte $\mathrm{J}$ - Effects of temperature on phrenic nerve and diaphragmatic function during cardiac surgery. Br H Anaesth 1997; 79: 726-32.
10 Setina M, Cerny S, Grim M, Pirk J - Anatomical interrelation between the phrenic nerve and the internal mammary artery as seen by the surgeon. $J$ Cardiovasc Surg (Torino) 1993; 34: 499-502.

11 Sahn S A \& Lakshminarayan S - Bedside criteria to discontinuation of mechanical ventilation. Chest 1973; 63: $1002-5$.

12 Yang $\mathrm{K}$ \& Tobin M J - A prospective study of indexes predicting the outcome of trials of weaning from mechanical ventilation. N Engl J Med 1991; 324: 1445-50.

13 Fiaccadori E, Del Canale S, Vitali P, Coffrini E, Ronda $\mathrm{N}$, Guariglia A - Skeletal muscle energetics, acidbase equilibrium and lactate metabolism in patients with severe hypercapnia and hypoxemia. Chest 1987; 92: 883-7.

14 Fiaccadori E, Del Canale S, Coffrini E et al. - Muscle and serum magnesium in pulmonary intensive care unit patients. Crit Care Med 1988; 16: 751-60.

15 Fiaccadori E, Coffrini E, Fracchia C, Rampulla C, Montagna T, Borghetti A - Hypophosphatemia and phosphorus depletion in respiratory and peripheral muscles of patients with respiratory failure due to COPD. Chest 1994; 105: 1392-8.

16 Tarasoutchi F, Auler Jr. J O C, Dallan L A O et al. Paralisia bilateral do diafragma no pós-operatório imediato de cirurgia cardíaca. Arq Bras Cardiol 1990; 55: 51-3.

17 Fell S C - Surgical anatomy of the diaphragm and the phrenic nerve. Chest Surg Clin N Am 1998; 8: 281-94.

18 Creasey G, Elefteriades J, Di Marco A et al. - Electrical stimulation to restore respiration. J Rehabil Res Dev 1996; 33: 123-32. 